LHC-B/96-5

$\mathrm{RICH} / 96-1$

R.W. Forty

\title{
Ring-Imaging Cherenkov Detectors for LHC-B
}

\author{
Roger Forty ${ }^{1}$ \\ CERN, Geneva
}

\begin{abstract}
The particle identification system proposed for the LHC-B experiment is presented. It consists of ring-imaging Cherenkov detectors with three radiator materials, including the novel use of aerogel in an imaging detector. The photodetectors under development are multipixel hybrid photodiodes, which will allow high performance to be achieved due to their excellent single-photon efficiency and high spatial resolution. Significant $\pi / K$ separation will be possible for isolated tracks with momenta between 1 and $150 \mathrm{GeV} / c$; the first studies of pattern recognition in regions of high track density are described.
\end{abstract}

Invited talk at BEAUTY 96, 4th Int. Workshop on B-physics at Hadron Machines Rome, 17-21 June 1996, to appear in Nucl. Instrum. Meth.

\footnotetext{
${ }^{1}$ Roger.Forty@cern.ch
} 



\section{Introduction}

LHC-B is a collider-mode forward spectrometer experiment, designed for the study of CP violation in $B$ decays. It is intended to be ready right at the start of the LHC's operation; however, as that will not be until the middle of the next decade, evidence of $\mathrm{CP}$ violation in $B$ decays may already have been seen at other machines (such as HERA or the $e^{+} e^{-}$ $B$ factories). It is thus a "second generation" experiment, intended to reach the ultimate precision in the detailed study of CP-violating asymmetries for many channels, and will therefore require careful control of systematic errors. For this, particle identification is crucial.

In high-energy $p p$ collisions the production of $b$ hadrons is expected to be predominantly in the forward direction, so the LHC-B spectrometer covers the region of polar angle $\theta<400 \mathrm{mrad}$. Furthermore, a strong correlation is predicted between track angle and momentum, as illustrated in Fig. 1, with forward tracks having a harder momentum spectrum. The requirements for particle identification can be determined from the momentum spectra of tracks from representative low- and high-multiplicity decays, $B_{d}^{0} \rightarrow \pi^{+} \pi^{-}$and $B_{s}^{0} \rightarrow D_{s}^{-} \pi^{+} \pi^{+} \pi^{-}$. The low-multiplicity decays define the upper momentum limit required for $\pi / K$ separation: in about $90 \%$ of $B_{d}^{0} \rightarrow \pi^{+} \pi^{-}$decays neither track has momentum greater than $150 \mathrm{GeV} / c$ in the very forward region $(13<\theta<120 \mathrm{mrad})$, or greater than $65 \mathrm{GeV} / c$ over the rest of the acceptance. The high-multiplicity decays define the lower momentum limit: in about $90 \%$ of the $B_{s}^{0} \rightarrow D_{s}^{-} \pi^{+} \pi^{+} \pi^{-}$decays (with a six-track final state) none of the tracks have momentum less than $1 \mathrm{GeV} / c$ over the whole acceptance. Thus we wish to separate pions from kaons unambiguously over the momentum range $1<p<65 \mathrm{GeV} / c$, and up to $150 \mathrm{GeV} / c$ in the very forward region.

These requirements can be met by ring-imaging Cherenkov (RICH) detectors, with three different radiator materials to cover the different momentum regions: two gases $\left(\mathrm{CF}_{4}\right.$ and $\left.\mathrm{C}_{4} \mathrm{~F}_{10}\right)$, and aerogel. Some properties of these materials (including aerogel with two different refractive indices) are listed in Table 1. The layout of the proposed RICH system is described in the next section.

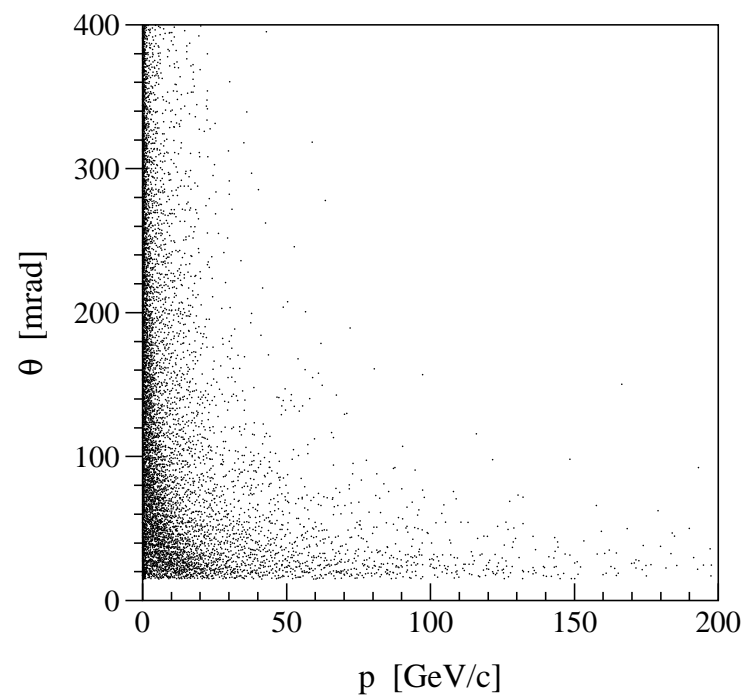

Figure 1: Polar angle $\theta$ vs. momentum $p$ for tracks in $B$ events (from the PYTHIA Monte Carlo); the cut at small angle is due to the beam pipe. 


\begin{tabular}{|lr|r|r|r|r|}
\hline \multicolumn{2}{|l|}{ Material } & & $\mathrm{CF}_{4}$ & $\mathrm{C}_{4} \mathrm{~F}_{10}$ & \multicolumn{2}{|c|}{ Aerogel } \\
\hline$n$ & & 1.0005 & 1.0014 & 1.03 & 1.06 \\
$\theta_{\mathrm{c}}^{\text {max }}$ & {$[\mathrm{mrad}]$} & 30 & 53 & 240 & 340 \\
$p_{\text {thresh }}(\pi)$ & {$[\mathrm{GeV} / c]$} & 4.6 & 2.7 & 0.6 & 0.4 \\
$p_{\text {thresh }}(K)$ & {$[\mathrm{GeV} / c]$} & 16.3 & 9.4 & 2.0 & 1.4 \\
\hline$\sigma_{\theta}^{\text {emission }}$ & {$[\mathrm{mrad}]$} & 0.1 & 0.6 & 0.3 & 0.5 \\
$\sigma_{\theta}^{\text {chromatic }}$ & {$[\mathrm{mrad}]$} & 0.3 & 0.6 & 1.2 & 1.7 \\
$\sigma_{\theta}^{\text {pixel }}$ & {$[\mathrm{mrad}]$} & 0.2 & 0.5 & 0.5 & 0.5 \\
$\sigma_{\theta}^{\text {total }}$ & {$[\mathrm{mrad}]$} & 0.4 & 1.0 & 1.4 & 1.9 \\
$N_{\text {pe }}$ & & 26 & 55 & 15 & 28 \\
$\sigma_{\theta}^{\text {track }}$ & {$[\mathrm{mrad}]$} & 0.07 & 0.13 & 0.36 & 0.36 \\
$\sigma_{\beta}^{\text {track }}$ & $/ 10^{-6}$ & 2 & 7 & 88 & 127 \\
\hline
\end{tabular}

Table 1: Some characteristics of the radiator materials used in the RICH system; the lower part lists the contributions to the resolution (from emission-point, chromatic and pixel errors), the total resolution per photoelectron, the mean number of detected photoelectrons in the ring image, and the resolution per track on $\theta_{\mathrm{c}}$ and $\beta$, for the proposed RICH detectors.

\section{Optimized geometry}

A description of the LHC-B spectrometer can be found in Ref. [1, 2]. The region of approximately $2-7 \mathrm{~m}$ from the interaction point is occupied by a large dipole magnet, with many tracking stations to provide accurate momentum reconstruction. For low-momentum tracks, particle identification must occur upstream of the dipole, before they are swept out of the acceptance. On the other hand, the very forward region is best instrumented downstream of the dipole, where the track separation is greater. The proposed system therefore has two RICH stations, with the $\mathrm{CF}_{4}$ radiator (for high-momentum tracks) in a downstream station about $10 \mathrm{~m}$ from the interaction point. This detector is described in Ref. [1, 3], and features a gas radiator length of 1-2 $\mathrm{m}$ and a spherical focusing mirror with $12 \mathrm{~m}$ radius-of-curvature centred on the interaction point. A plane mirror is placed in front of the focusing mirror, inclined at $45^{\circ}$ to bring the image out of the LHC-B acceptance, so that the photodetector material does not disturb the detectors that follow (calorimeters and muon chambers); the particle flux through the photodetectors is also substantially reduced.

The original design for the upstream station [1, 3] consisted of two consecutive detectors, the first with aerogel radiator and the second with a high-index gas, and each with a geometry similar to that of the $\mathrm{CF}_{4}$ detector. This had some disadvantages: as the aerogel was up against the focusing mirror, any Cherenkov light produced had to traverse the full thickness of the aerogel before reaching the detector; as discussed in Section 3, this leads to a significant reduction of the number of photons, due to scattering. Secondly, the gas radiator length was constrained by the need to fit both the aerogel counter and angled mirror in the limited space between the vertex detector and the spectrometer magnet.

To avoid these drawbacks, a new geometry has been adopted for the upstream RICH station, which combines both aerogel and gas radiators in the same device, as illustrated in Fig. 2. The aerogel is moved up to the entrance window, so that light is now produced in transmission; the spherical focusing mirrors (one for each half of the detector, with $2 \mathrm{~m}$ 


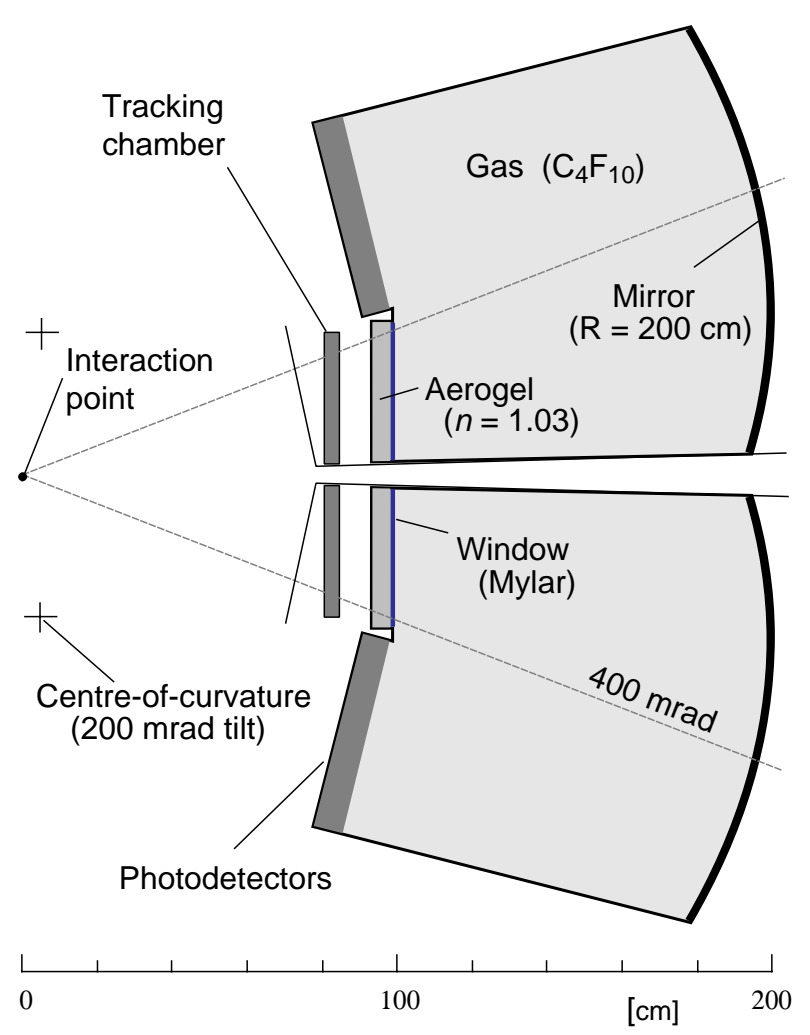

Figure 2: Layout of the new upstream RICH detector, seen from above (with the beam axis horizontal and the interaction point on the left-hand side).

radius of curvature) are tilted by about $200 \mathrm{mrad}$, to bring the image out of the acceptance; and the volume between the aerogel and mirrors is filled with $\mathrm{C}_{4} \mathrm{~F}_{10}$ gas. This is chosen due to its high refractive index and low dispersion; it is the heaviest fluorocarbon that remains gaseous at room temperature. As well as increasing the photon yield for both radiators, this layout has the advantage of almost halving the number of photodetectors required (as the same image plane is shared), and the amount of material is reduced as tracks pass through only a single mirror instead of four.

There were two critical issues that had to be addressed for this design: whether the shared image plane leads to problems for the pattern recognition (discussed in Section 5), and whether the tilted mirror introduces unacceptable aberrations to the ring image. This was studied using ray tracing: for a given simulated track, photons are generated at fixed polar and azimuthal Cherenkov angles $\left(\theta_{c}, \phi_{c}\right)$ along its length in the radiator. They are then reflected off the spherical mirror (initially aligned with centre-of-curvature at the interaction point), and their point of intersection found on a plane transverse to the beam axis. The position $z_{\text {min }}$ of this plane along the beam axis that minimizes the spread of impact points is then determined, and this is repeated for many azimuthal angles and many track impact points $(x, y)$ on the entrance window, to map the focal plane. The expected spherical focal surface is reproduced, with radius equal to half that of the mirror, as seen in Fig. 3 (a). When the mirror is tilted by $200 \mathrm{mrad}$, the result is shown in Fig. $3(\mathrm{~b})$ : the image moves out of the acceptance, as required, but the focal surface is no longer exactly spherical. For simplicity a planar photodetector surface is assumed, and the position of that plane is optimized to follow as closely as possible the focal surface: this leads to the angled detector plane visible in Fig. 2. The distribution of 


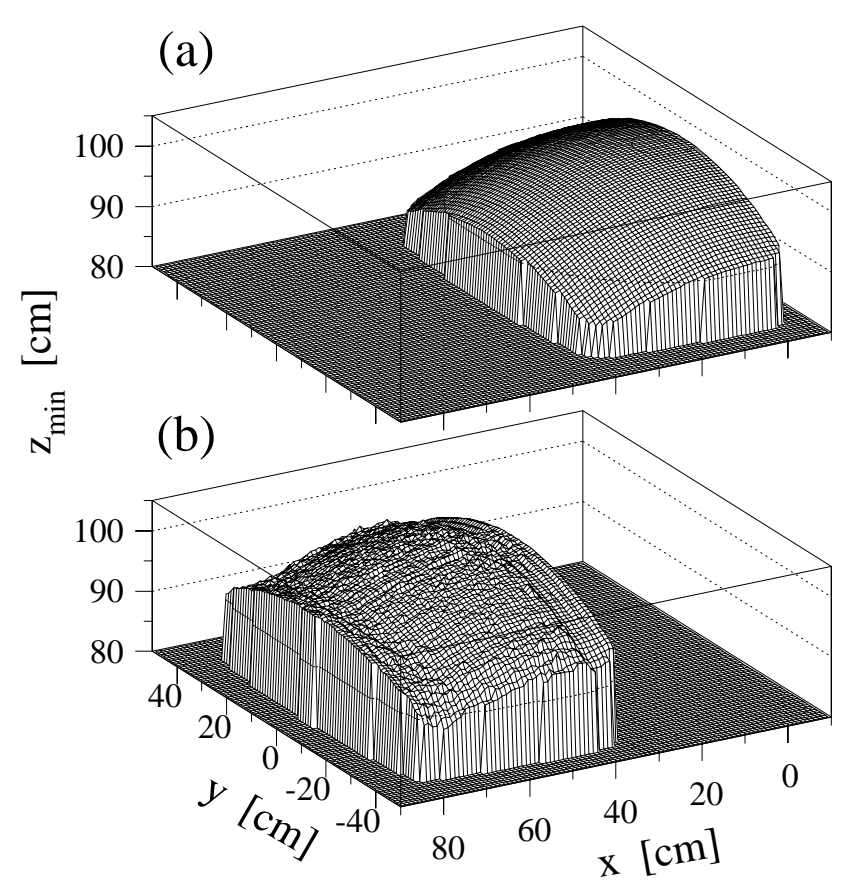

Figure 3: Reconstructed focal surface for a RICH: (a) with axial spherical mirror (with $2 \mathrm{~m}$ radius of curvature); (b) with mirror tilted by $200 \mathrm{mrad}$.

photon impact points on this plane, relative to their expected impact point for a perfectly imaging system, has an RMS of about $400 \mu \mathrm{m}$ in both projections (for the $\mathrm{C}_{4} \mathrm{~F}_{10}$ radiator).

To express this resolution in terms of its effect on the reconstructed Cherenkov angle $\theta_{c}$, which is the crucial issue for a RICH detector, it is necessary to determine the Cherenkov angles from the detected photon position and the assumed emission point (taken to be on the track, in the middle of the radiator). This is essentially the inverse of ray tracing, but is surprisingly difficult to solve for a spherical mirror; it is, however, important as it also provides the starting point for pattern recognition studies described in Section 5. The solution follows from realizing that the point of reflection lies on a plane defined by the points of emission and detection, and the centre-of-curvature $(\mathcal{C})$ of the mirror. Defining $s=\sin \alpha$, where $\alpha$ is the angle between the emission and reflection points in this plane $($ about $\mathcal{C})$, the requirement that the angle of reflection is bisected by the normal to the mirror surface leads to a quartic equation:

$$
\begin{aligned}
4 a^{2}\left(b^{2}+c^{2}\right) s^{4} & -4 a^{2} c R s^{3}+\left(c^{2} R^{2}+(a+b)^{2} R^{2}-4 a\left(b^{2}+c^{2}\right) R\right) s^{2} \\
& +2 a c(a-b) R s+\left(a^{2}-R^{2}\right) c^{2}=0
\end{aligned}
$$

where $a$ is the length of the vector from $\mathcal{C}$ to the emission point, $b$ and $c$ are the components, parallel and orthogonal to that vector, of the distance from $\mathcal{C}$ to the detection point, and $R$ is the radius of curvature of the mirror. This gives four solutions for $s$, two complex and two real, and of the real solutions one is the "backward" reflection (that would exist if the mirror were a complete sphere); the other is the desired solution. ${ }^{2}$

Using this procedure, the contribution to the resolution on $\theta_{c}$ from the imperfect focusing of the tilted mirror (or equivalently, from the uncertainty on the photon emission point) is shown in Fig. 4 (a), with an RMS of $0.6 \mathrm{mrad}$. This is not a dominant contribution

\footnotetext{
${ }^{2}$ This calculation gives equivalent results to the analysis of Ref. [4], despite the different approach.
} 


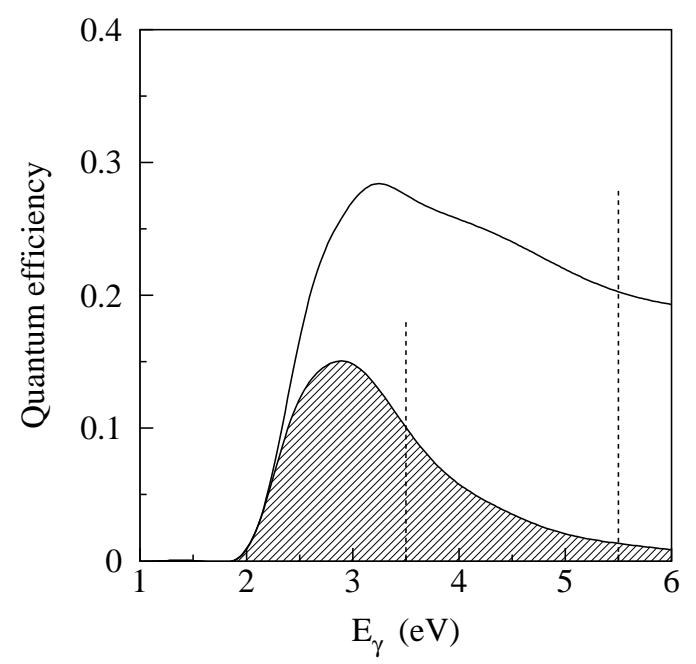

Figure 5: Quantum efficiency assumed for the photodetectors as a function of the incident photon energy; the shaded distribution shows the effective quantum efficiency for Cherenkov light produced in a sample of aerogel, taking Raleigh scattering into account, and the dashed lines indicate the assumed window cut-offs for the two distributions.

\section{Aerogel}

Silica aerogel is a colloidal form of quartz, that is solid but very light. It has a longestablished use in threshold Cherenkov counters, but the idea of using it for an imaging detector is recent [5], and has followed from the development of high quality, very clear, samples. Its attraction is that it can be produced with refractive index in the range 1.01-1.10, suitable for the low momentum end of the LHC-B particle identification requirements, otherwise only available with pressurized gas.

The dominant cause of the scattering of light within aerogel is Raleigh scattering, with the result that the transmission of light with wavelength $\lambda$ through a block of thickness $L$ is proportional to $\mathrm{e}^{-C L / \lambda^{4}}$, where $C$ is a coefficient that characterizes the clarity of the sample; recent samples (with a refractive index of $\sim 1.03$ ) have $C \sim 0.01 \mu \mathrm{m}^{4} / \mathrm{cm}$, or even lower. This leads to $50 \%$ transmission for a $2 \mathrm{~cm}$ thick sample at about $400 \mathrm{~nm}$, with little transmission below $300 \mathrm{~nm}$.

The scattered photons are expected to emerge at any angle, and will therefore lead to a randomly distributed background on the image plane. Taking into account the production of Cherenkov light by a particle traversing a sample of aerogel (uniform along its length), and the scattering of that light, the fraction of produced photons that will survive unscattered is given by $\left(1-\mathrm{e}^{-C L / \lambda^{4}}\right) \lambda^{4} / C L$. For $L=5 \mathrm{~cm}$ and $C=0.01 \mu \mathrm{m}^{4} / \mathrm{cm}$, this leads to the effective quantum efficiency shown by the shaded distribution in Fig. 5 . The scattering dominates at high energy, so a thin window (of mylar, or glass) will be placed after the aerogel in the upstream RICH detector, to absorb the (mostly scattered) photons with $E_{\gamma}>3.5 \mathrm{eV}$. This also serves to reduce the chromatic aberration, and separate the aerogel from the gas. For a track passing through $5 \mathrm{~cm}$ of aerogel with $n=1.03$ the resulting number of detected photoelectrons in a saturated ring image is expected to be approximately 15, from Eq. (2), with an additional 5 or so scattered over the detector plane.

The contributions to the resolution have been determined for the aerogel radiator in the upstream $\mathrm{RICH}$, in a similar manner to those for the gas radiator described in the 


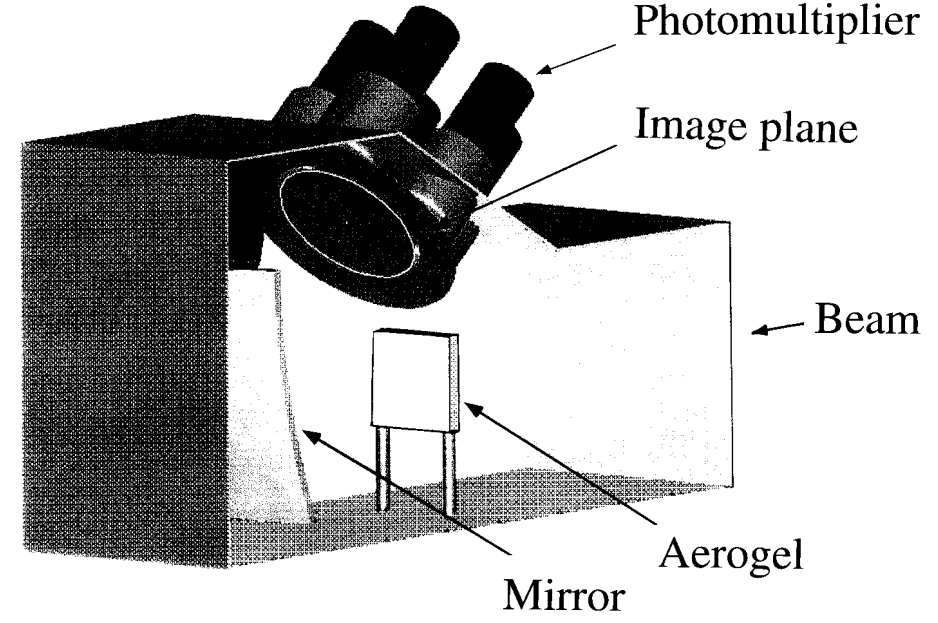

Figure 6: Apparatus used for the aerogel beam test; (for the test described, the illustrated photomultipliers were replaced by a one-inch tube, mounted on a motorized stage).

previous section. The results are compared in the lower part of Table 1: the emissionpoint contribution is reduced (due to the smaller radiator thickness), the chromatic error is greater (due to the higher dispersion), and the pixel error is unchanged. The overall resolution per photoelectron is $1.4 \mathrm{mrad}$, which is reasonably well matched to the gas radiator resolution, permitting the use of common photodetectors. Also shown in the table are the equivalent figures for aerogel with a higher refractive index: the resolution per photoelectron is poorer, but this is offset by the larger number of photoelectrons per track, so the resolution per track on $\theta_{\mathrm{c}}$ is unchanged. However, expressing the resolution in terms of the particle velocity $\beta$, which determines the particle-identification performance, the higher index sample gives a poorer $\sigma_{\beta}$, since $\sigma_{\beta} \approx \theta_{\mathrm{c}} \sigma_{\theta}$ (for small $\theta_{\mathrm{c}}$ ); the $n=1.03$ aerogel is therefore favoured, although the final choice will also depend on pattern-recognition considerations.

A beam test of aerogel is currently underway at CERN, by LHC-B in collaboration with groups from Bari, Lecce and Rome (Sanità) ${ }^{3}$. The apparatus used is illustrated in Fig. 6: it consists of a light-tight box, flushed with nitrogen, containing an angled spherical mirror (of $90 \mathrm{~cm}$ radius-of-curvature) with a sample of aerogel supported in front; a one-inch photomultiplier mounted on a motorized stage is arranged so that it can scan horizontally across the focal plane of the mirror. This setup has been exposed to a $10 \mathrm{GeV} \pi^{-}$beam from the CERN PS. The results presented here are from a $3 \mathrm{~cm}$ thick sample of aerogel produced at KEK, with nominal refactive index $n=1.029$ and measured clarity $C=0.01 \mu \mathrm{m}^{4} / \mathrm{cm}$.

The passage of pions through the nitrogen gas generates Cherenkov light at small angle ( $\sim 20 \mathrm{mrad})$, leading to a ring on the focal plane that is not resolved by the photomultiplier. A threshold was applied to the photomultiplier output to suppress noise but maintain sensitivity to single photoelectrons, and it was then scanned across the image plane. The variation of the number of counts (per $10^{4}$ triggers) with position $r$ is shown in Fig. 7, where the origin of position has been chosen to lie at the centre of the strong nitrogen signal. A clear peak is seen in the count rate at $r=11.6 \mathrm{~cm}$, corresponding to the Cherenkov ring image from the aerogel (the same enhancement was also seen when

\footnotetext{
${ }^{3}$ These groups are planning to use aerogel in an upgrade of the HERMES experiment at DESY; their help in providing and setting up the detector is gratefully acknowledged.
} 


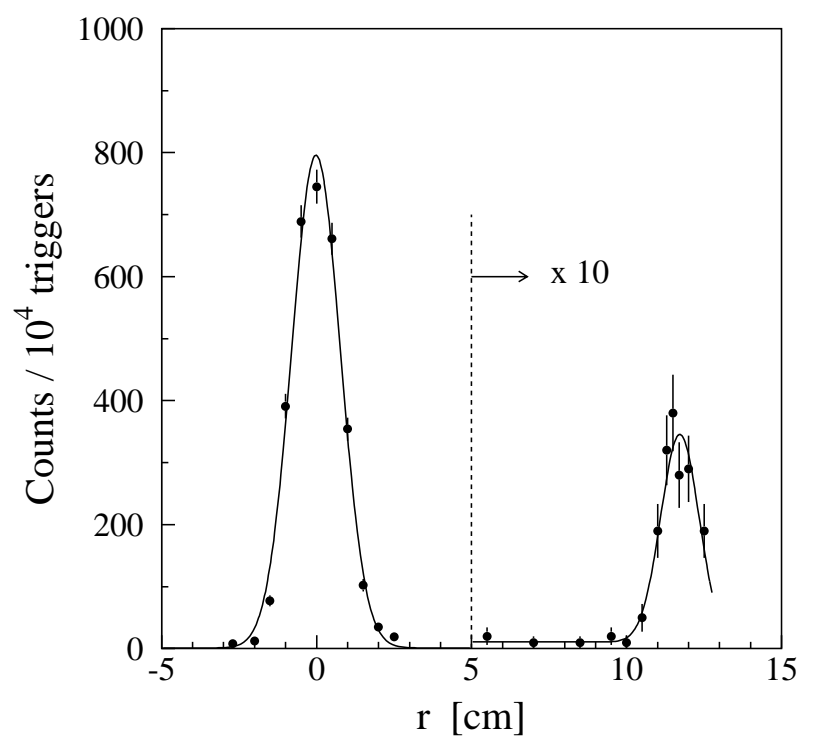

Figure 7: Number of photomultiplier counts as a function of the position of the photomultiplier across the image plane, in the aerogel beam test; the peak around $r=0$ is from the nitrogen ring, the other is from the aerogel.

the scan was made in the opposite direction); the width of the peak is dominated by the size of the photomultiplier. Given the focal length of the mirror, this radius corresponds to a refractive index of 1.03 , consistent with its nominal value. The background count rate, between the aerogel and nitrogen peaks, is very low, as expected for the high clarity aerogel sample.

The pulse-height distribution from the photomultiplier is shown in Fig. 8. When the photomultiplier is positioned on the aerogel peak (open histogram) the one- and twophotoelectron peaks are clearly seen; in the background region (at $r=6 \mathrm{~cm}$, shaded) there is some tail above the pedestal, which includes the contribution from Raleigh scattered

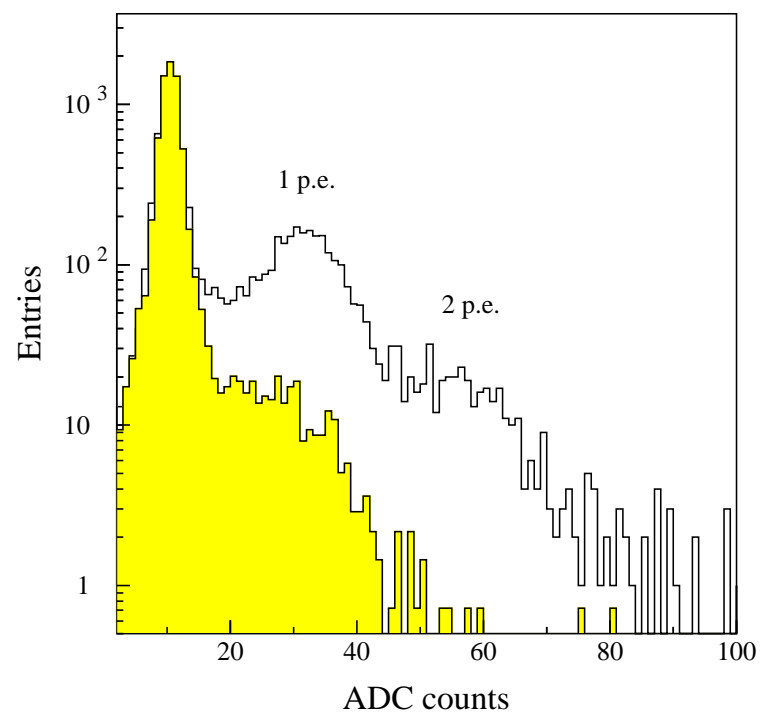

Figure 8: Pulse-height spectrum for the photomultiplier, when positioned on the aerogel peak (open histogram) and off-peak (shaded), where the latter has been scaled to match the pedestal of the on-peak distribution. 
photons. Scaling the latter distribution so that its pedestal matches that of the former, the fraction of events with no signal can be determined when on the aerogel peak: $f_{0}=0.70$. For a Poisson distribution, $f_{0}=\mathrm{e}^{-\mu}$, where $\mu$ is the mean number of photoelectrons detected within the photomultiplier acceptance; thus $\mu=0.35$. Scaling by the ratio of the aerogel ring circumference to the photomultiplier diameter, this corresponds to about 14 detected photoelectrons per track, which is in good agreement with the expectation. ${ }^{4}$

\section{Photodetectors}

The requirements for the photodetectors of the LHC-B RICH system are the following:

1. Single photelectron sensitivity (for the aerogel high quantum efficiency is required in the visible);

2. Fast enough for LHC (where the time between bunch crossings is $25 \mathrm{~ns}$ );

3. Pixel size $2 \times 2 \mathrm{~mm}^{2}\left(4 \times 4 \mathrm{~mm}^{2}\right.$ for the downstream detector $)$;

4. Low noise;

5. Large area coverage $\sim 1.2 \mathrm{~m}^{2}\left(2.6 \mathrm{~m}^{2}\right.$ for the downstream detector $)$, with highest possible active area.

Assuming that the devices are cylindrical with $10 \mathrm{~cm}$ diameter, the required area corresponds to 140 units (300 for the downstream detector). Hexagonal close-packing gives $90 \%$ coverage, and assuming an $80 \%$ active area within the device, this leads to 200,000 pixels in the upstream detector (120,000 in the downstream). Low cost per pixel is therefore essential.

These requirements are not all met by any currently available detector, so a vigorous programme of R\&D is underway. The main focus is on hybrid photodiodes (HPD's), which involve the electrostatic acceleration of electrons from a photocathode into a silicon detector [6]. Such devices are available commercially with a few pixels (and small active area), with a feed-through for each pixel out of the vacuum envelope; the main challenge is to increase the number of pixels per detector to $\mathcal{O}(1000)$, necessary to achieve the desired ratio of active to total area. In this case a feed-through per channel becomes impractical and it is necessary to include some electronics within the vacuum envelope. Two approaches are pursued:

1. Strongly focussed, so that the photocathode is imaged onto a small detector with $\mathcal{O}(100 \mu \mathrm{m})$ pixels, bump-bonded to a readout chip with matching pixel electronics;

2. Proximity focussed (or only gently focussed), onto a larger detector with $\mathcal{O}(1 \mathrm{~mm})$ pads, read out via conductive tracks on the silicon surface to a separate electronics chip.

The first approach is represented by the "Imaging Silicon Pixel Array" (ISPA tube), for which a prototype exists with 1024 pixels of $500 \times 75 \mu \mathrm{m}^{2}$, and an $18 \mathrm{~mm}$ diameter

\footnotetext{
${ }^{4}$ The prediction of 15 detected photoelectrons given earlier was for a thicker sample $(5 \mathrm{~cm})$, but only $70 \%$ detector coverage was assumed.
} 

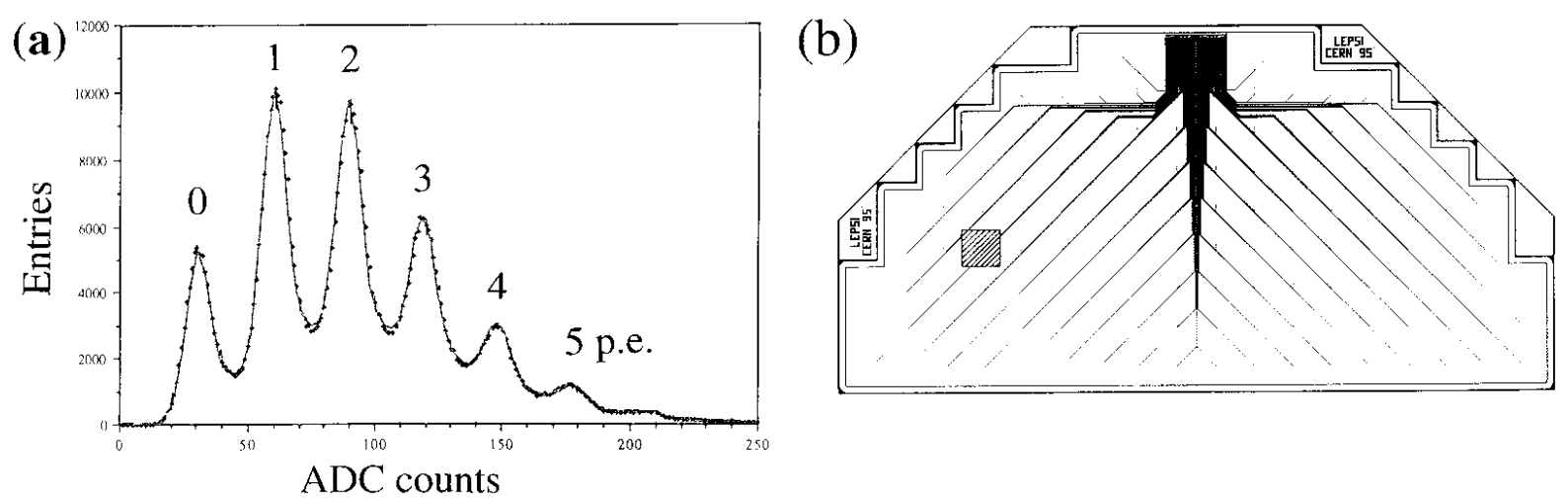

Figure 9: (a) Pulse-height spectrum from the global-analogue output of the ISPA tube, illuminated with a low-intensity LED; the left-most peak is the pedestal, the others show the clearly resolved photoelectron signals; (b) layout of a silicon pad detector: one of the $4 \times 4 \mathrm{~mm}^{2}$ pads is shaded for clarity; the tree-like structure visible is the fanin to the electronics chip, which is wire-bonded to the upper edge of the wafer.

photocathode [7]. It was produced by an RD-7/LAA collaboration, using pixel electronics developed by RD-19 [8], encapsulated in a vacuum envelope by DEP (Netherlands). The single-photoelectron efficiency of this detector was recently calibrated [9] using a lowintensity LED that gave the pulse-height spectrum shown in Fig. 9 (a), where the signal from a global analogue output is shown, from the backplane of the silicon. The individual photoelectron peaks are clearly resolved, and a fit to the spectrum gives a mean number of 2.4 photoelectrons/event. The fit also requires a term due to backscattering [10] from the silicon, which leads to a tail below each peak (and therefore fills in between the peaks), with a backscattering probability of 15-20\%. Next the number of fired pixels was counted, and gave an average of 1.6 hits/event at an accelerating voltage of $27 \mathrm{kV}$. After correcting for the backscattering, the efficiency is thus about $80 \%$. The reduction from full efficiency is almost all accounted for by charge sharing between the pixels: this brings the charge per pixel below the relatively high threshold that is applied in the current version of the pixel electronics [8]; the effect of charge sharing will be negligible for the larger pixels that will eventually be used. At lower voltages the efficiency is reduced, falling off below $20 \mathrm{kV}$, due to the high threshold. The next step is under design, using a new pixel chip [11], with lower threshold and compatable with LHC speeds. A large photocathode will be used, with active diameter $80 \mathrm{~mm}$, and a demagnifying factor of $\sim 4$ onto the silicon. Tests have been made using an image-intensifier with similar focussing in a magnetic field [9]. For 40 Gauss (the fringe-field expected from the LHC-B spectrometer magnet) the observed distortions are small, less than the equivalent of $500 \mu \mathrm{m}$ at the photocathode.

The second approach, using gentle focussing onto a pad detector, is described in detail in an accompanying contribution [12]. Silicon half-wafers implanted with $1284 \times 4 \mathrm{~mm}^{2}$ pads have been produced, with the layout shown in Fig. 9 (b). The fanin brings the signals to the edge of the wafer, where they are connected to a VA2 readout chip by wire bonding. Pairs of these detectors have been mounted in a test chamber, pumped for vacuum, with a CsI photocathode and $15 \mathrm{kV}$ accelerating voltage, illuminated by a collimated light source. The first results are very promising, with low noise $\left(\sim 300 e^{-}\right.$ ENC) and cleanly separated photoelectron peaks [12]. The next step for this development is to use smaller pads $\left(1 \times 1 \mathrm{~mm}^{2}\right)$, with LHC-compatable electronics, and encapsulate 
the detector in a glass tube under vacuum to form a prototype device.

\section{Pattern recognition}

A typical simulated $B$ event in the upstream RICH is shown in Fig. 10. Here the two detector planes are drawn side-by-side, and crosses mark the impact points of the charged tracks in the event, extrapolated to the detectors as if they were reflected by the mirror. Dots mark the positions of detected photoelectron "hits" (assuming the quantum efficiency discussed earlier). Those originating from the $\mathrm{C}_{4} \mathrm{~F}_{10}$ gas radiator are visible as well-defined rings, of about $10 \mathrm{~cm}$ maximum diameter. The aerogel radiator leads to larger, more sparsely-populated rings, that are less obvious to the eye: one is picked out by circling its hits in the figure. There are also background hits from Raleigh scattering in the aerogel, that are not associated to any ring. The event generator is PYTHIA, and a full GEANT treatment is used to provide track impact points on the entrance window of the RICH; a stand-alone routine is then used to simulate the Cherenkov light.

As can be seen for the gas radiator, the Cherenkov rings are not perfect circles, but are rather elliptical in shape, with a degree of distortion that depends on the direction of

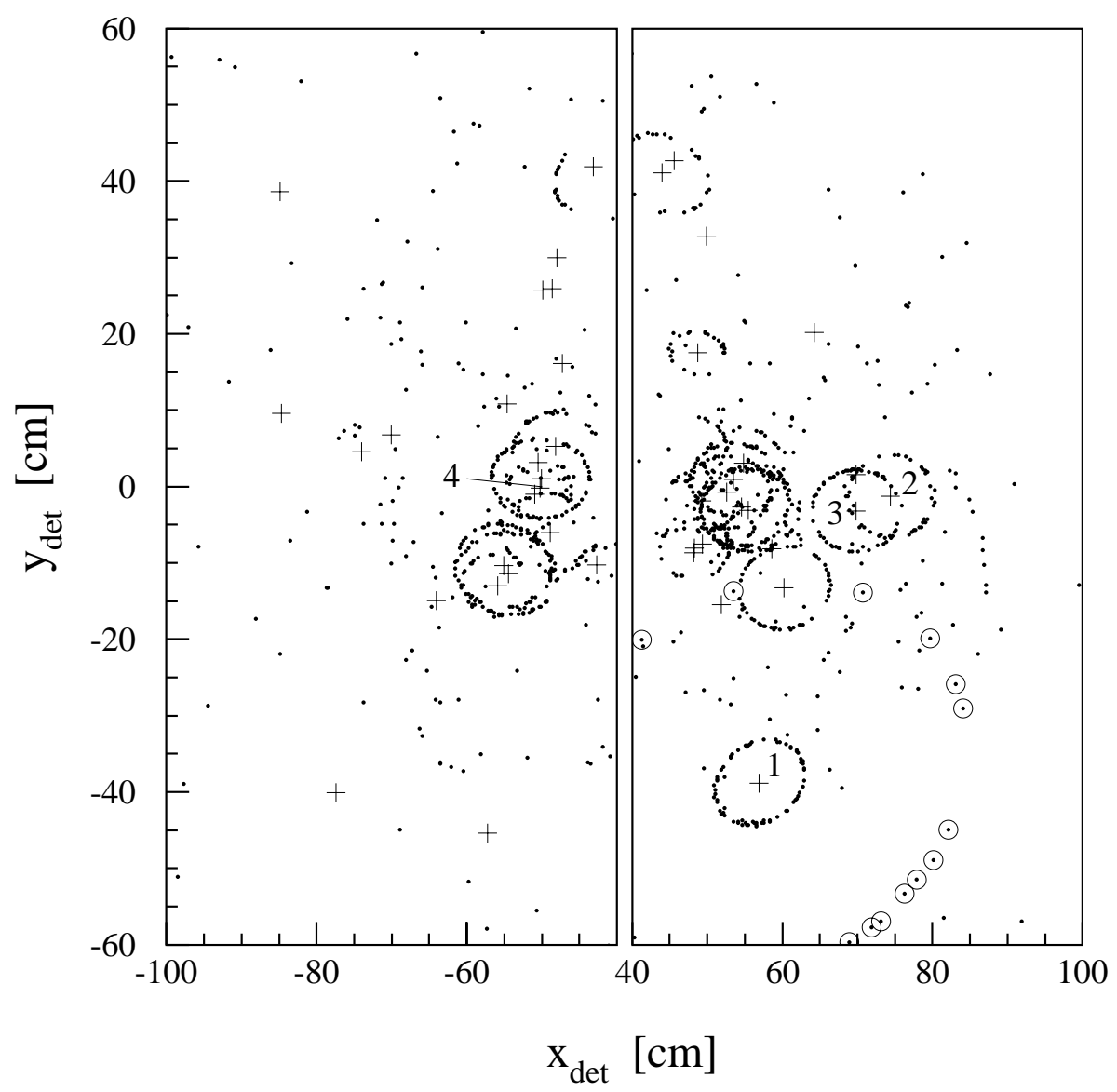

Figure 10: Display of the data from the upstream RICH for a typical simulated $B$ event: the two detector planes are drawn side-by-side for clarity; the dots represent detected photoelectrons, and the crosses are the impact points of the charged tracks (if they were reflected in the mirror), four of which are labelled; the hits on the aerogel ring image from Track 1 are circled for emphasis. 


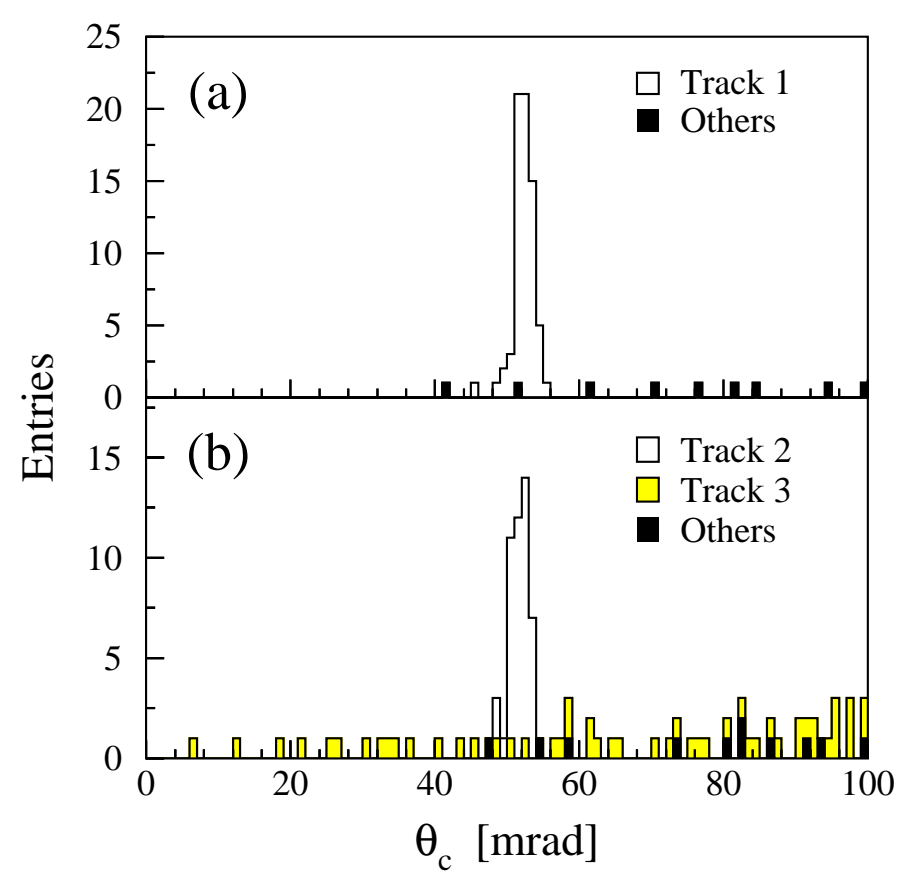

Figure 11: Reconstructed Cherenkov angle $\theta_{c}$ for hits in the event of Fig. 10: (a) assuming that the hits originate from Track 1; (b) assuming that they originate from Track 2; the true origin of the hits is indicated by shading.

the track within the acceptance. Instead of attempting to fit directly these rings, a great simplification is achieved by reconstructing the Cherenkov angles at emission, $\left(\theta_{\mathrm{c}}, \phi_{\mathrm{c}}\right)$, as described in Section 2. That calculation uses the hit position and the mirror parameters, and also the assumed photon emission point, which is taken to be on a track, half-way through the radiator; it is therefore made under the assumption that the hit originates from a given track. The hits which truly originate from that track will then all have the same value of polar Cherenkov angle $\theta_{i}=\theta_{\mathrm{c}}$ (within the resolution), and have uniformly distributed azimuthal angle $\phi_{i}$ (where the subscript $i$ denotes the assumption of parent track that has been made in calculating the angles).

This is illustrated in Fig. 11 (a), where the Cherenkov angle is reconstructed for the hits in the event assuming they originated from Track 1 (an isolated track, labelled in Fig. 10). A peak is seen at $\theta_{1}=52 \mathrm{mrad}$, the true Cherenkov angle for this track in the gas radiator; the few other entries in the histogram are scattered hits from the aerogel. In Fig. 11 (b) the same plot is made, but assuming the hits originate from Track 2, for which there is a close neighbour (Track 3). In this case, a clear peak is again seen at the correct $\theta$, but now there are more "background" hits, due to the signal from the overlapping ring of Track 3. This is clear in the two-dimensional plot of $\left(\theta_{2}, \phi_{2}\right)$ shown in Fig. 12: the hits from Track 3 describe a curling trajectory on this plot (whilst if $\left(\theta_{3}, \phi_{3}\right)$ is plotted they fall at constant $\theta$, and it is the hits from Track 2 that follow a similar curve).

The task of the pattern recognition is to identify such signals, even for tracks in the densely-populated regions of the event. An example is Track 4 in Fig. 10, for which the reconstructed $\theta$ plot is shown in Fig. 13 (a). Clearly most of the "background" to the signal of Track 4 (from the gas radiator) is in fact from signals of other tracks. Two approaches to pattern recognition have been pursued:

1. Search for the most significant peak. For each track the $\theta_{i}$ histogram is filled, 


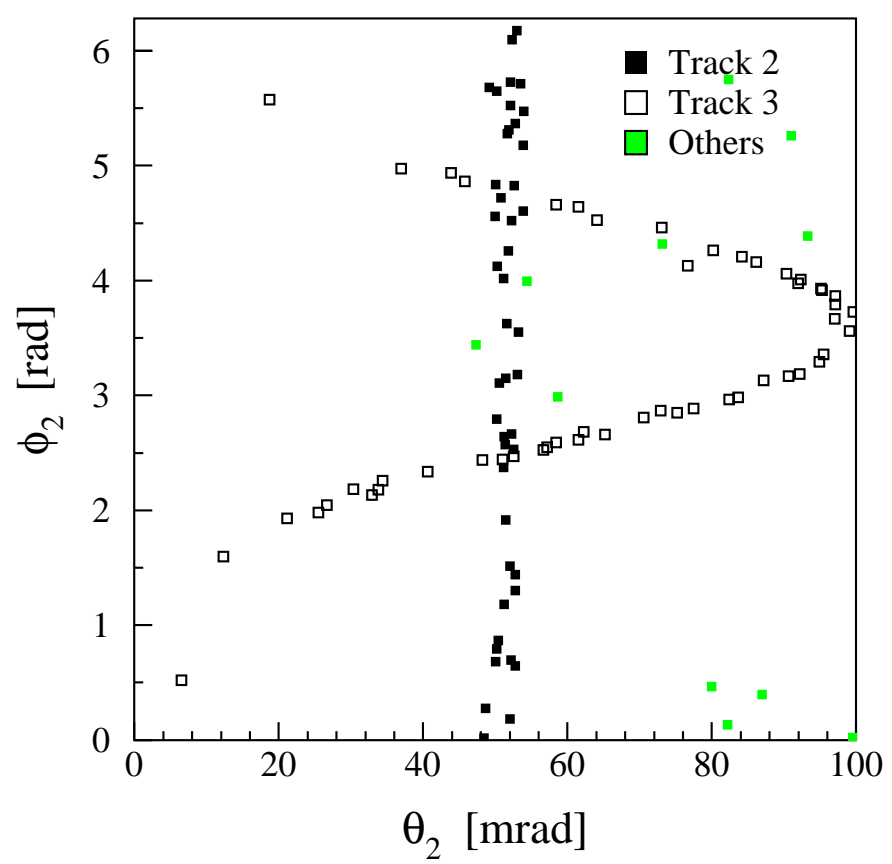

Figure 12: Reconstructed Cherenkov angles $\phi_{\mathrm{c}}$ vs. $\theta_{\mathrm{c}}$ for hits in the event of Fig. 10, assuming that they originate from Track 2 .

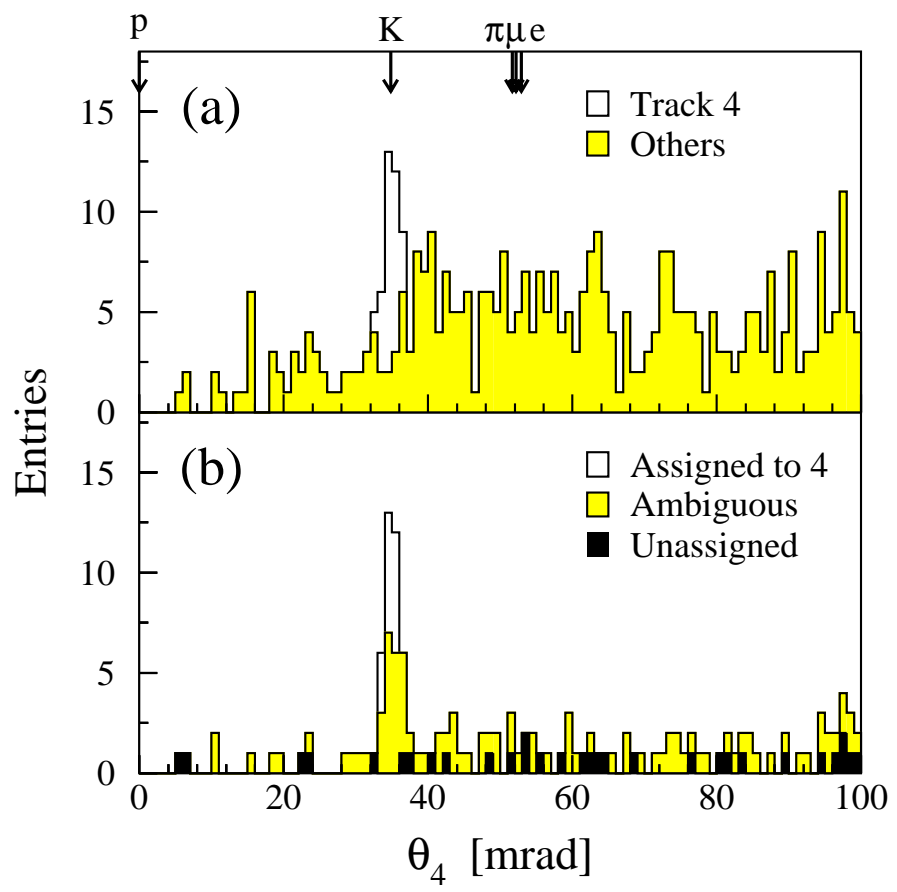

Figure 13: Reconstructed Cherenkov angle $\theta_{\mathrm{c}}$ for hits in the event of Fig. 10: (a) assuming that the hits originate from Track 4; the arrows indicate the expected signal positions for different particle types; (b) after removing the hits unambiguously assigned to other tracks. 
and a window is opened $\left( \pm 2 \sigma_{\theta}\right)$ around the expected $\theta_{\mathrm{c}}$ for each of the possible mass-hypotheses $(e, \mu, \pi, K, p)$. The number of entries within the window is counted, the background estimated from the side-bands, and the signal significance calculated. The most significant peak is selected, amongst all combinations of track and mass-hypothesis; the hits within that peak are flagged as assigned (and are not used in subsequent iterations), and the procedure is repeated for the remaining tracks. In the event illustrated, Track 1 has the most significant peak (under the $e$ hypothesis), whilst Track 4 has one of the lower significances; it is still, however, correctly identified (as a kaon), as are all tracks in the event. Removing the hits that have been unambiguously assigned to other tracks, the peak in the plot of $\theta_{4}$ becomes much cleaner, as shown in Fig. $13(\mathrm{~b})$. However, fitting such resulting $\theta_{i}$ distributions is no longer useful as they are biassed by the $\pm 2 \sigma$ cut that has already been applied. This is the motivation for the alternative approach, a global fit:

2. Simultaneously fit for $\theta_{i}$ of all tracks. A $\chi^{2}$ is calculated that the detected hits originate from the set of tracks, each with a given assumed $\theta_{i}$; the hits are assigned to the nearest track image (i.e. to the track $i$ that minimizes $\left|\theta_{\text {hit }}-\theta_{i}\right|$ ). Then:

$$
\chi^{2}=\sum_{\text {hit }} \frac{\left(\theta_{\text {hit }}-\theta_{i}\right)^{2}}{\sigma_{\theta}^{2}}+\sum_{\text {track }} \frac{\left(n_{\text {assign }}-n_{\text {expect }}\right)^{2}}{n_{\text {expect }}},
$$

where $n_{\text {assign }}$ is the number of hits assigned to a track, and $n_{\text {expect }}$ is the number expected (from Eq. (2)). To this $\chi^{2}$ a term is added for the flatness of the $\phi_{i}$ distributions, calculated from histograms of $\Delta \phi$ (the difference in $\phi_{i}$ for each pair of hits associated to a track) which are flat if the hits are truly from the same track. The $\chi^{2}$ is then minimized with respect to all $\theta_{i}$. For the event shown all tracks have their Cherenkov angle correctly reconstructed (within errors); the results for the four labelled tracks are given in Table 2.

The global approach looks promising; its extension to include the aerogel hits (with their poorer signal/background) is underway.

\begin{tabular}{|r|r|r|r|r|}
\hline Track & $\theta_{\text {true }}$ & $\theta_{\text {fit }}[\mathrm{mrad}]$ & $n_{\text {true }}$ & $n_{\text {assign }}$ \\
\hline 1 & 52.38 & $52.33 \pm 0.18$ & 45 & 45 \\
2 & 51.58 & $51.49 \pm 0.15$ & 66 & 64 \\
3 & 52.50 & $52.72 \pm 0.18$ & 45 & 47 \\
4 & 34.78 & $34.67 \pm 0.21$ & 29 & 32 \\
\hline
\end{tabular}

Table 2: Illustration of the global fit: the result for selected tracks from the event displayed in Fig 10. 


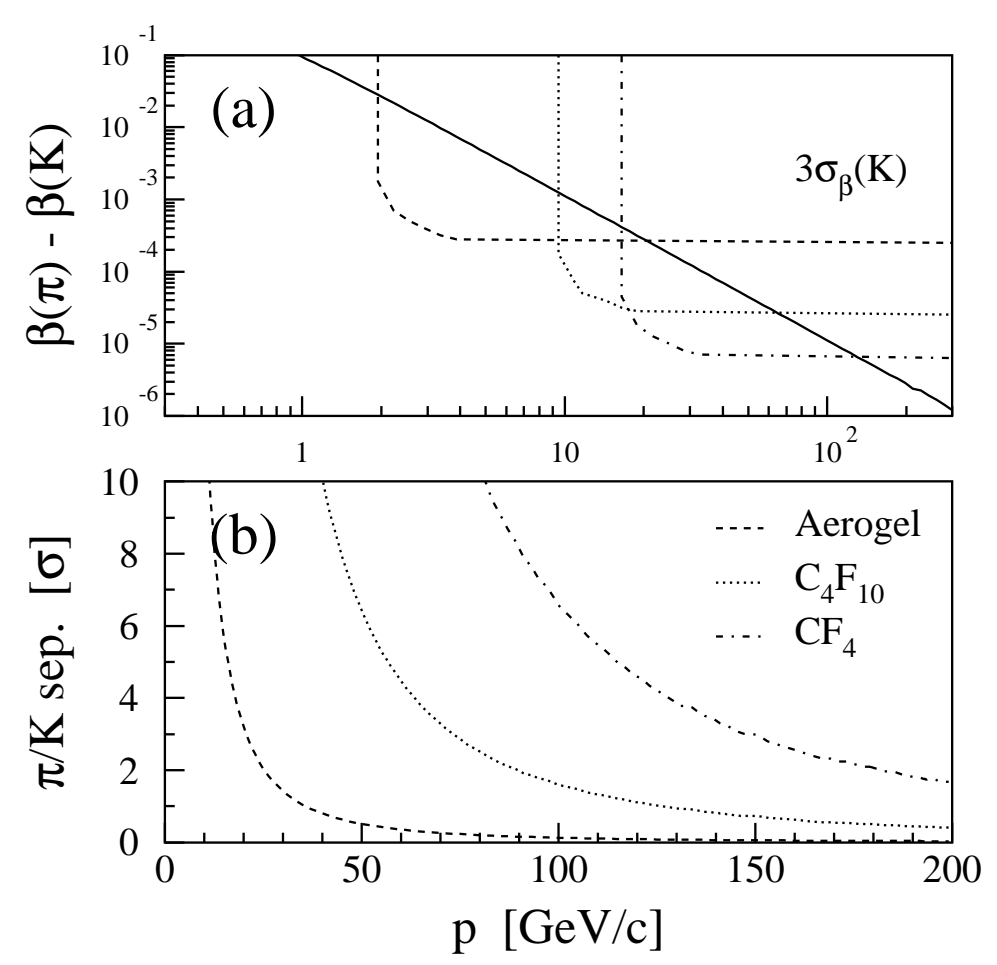

Figure 14: Particle-identification performance of the system: (a) the difference in velocity $\beta$ for the pion or kaon mass-hypothesis as a function of the track momentum; superimposed as dashed lines are the $3 \sigma$ resolutions on $\beta$ for kaons in the three radiator materials; (b) the significance of the $\pi / K$ separation in standard deviations, as a function of momentum, for the upper limit of identification in the three radiator materials.

\section{Conclusion}

The RICH detector system proposed for the LHC-B experiment has been described. The particle identification performance is illustrated in Fig. $14(\mathrm{a})$, where the difference in velocity $\beta$ for the pion or kaon mass-hypothesis is shown as a function of the track momentum. Superimposed is the $3 \sigma$ resolution on $\beta$ from each of the three radiators. The resulting significance of $\pi / K$ separation is shown in Fig. 14 (b) for the upper limit of identification in the three radiator materials (the lower limits are given by the momentum thresholds in Table 1). Between them the three radiators cover the full region $1<p<150 \mathrm{GeV} / c$ required. This is for isolated tracks; the degradation in denselypopulated regions is under study.

Beam tests of aerogel have demonstrated the formation of clean ring images, with little background from scattered light. HPD's are ideally matched to the photodetector requirements, and tests of multipixel prototypes are very promising; designs for the final implementation are well advanced. The simulation of the RICH system has allowed the geometry to be optimized, and studies of pattern recognition and potential backgrounds are underway. A prototype $\mathrm{RICH}$ with aerogel and $\mathrm{C}_{4} \mathrm{~F}_{10}$ gas radiators is under construction for beam test early next year, and we are confident that a realizable design for the final system can be defined for the LHC-B Technical Proposal by the end of 1997. 


\section{References}

[1] LHC-B Letter of Intent, "A dedicated LHC collider beauty experiment for precision measurements of CP violation", CERN-LHCC/95-5 (August 1995).

[2] G. Wilkinson (LHC-B Collaboration), "LHC-B overview", these proceedings, presented at BEAUTY 96, Rome (June 1996).

[3] T. Ypsilantis and J. Seguinot, Nucl. Instr. and Meth. A 368 (1995) 229.

[4] T. Ypsilantis and J. Seguinot, Nucl. Instr. and Meth. A 343 (1994) 30.

[5] D.E. Fields et al., Nucl. Instr. and Meth. A 349 (1994) 431.

[6] R. DeSalvo et al., Nucl. Instr. and Meth. A 315 (1992) 375.

[7] T. Gys et al., Nucl. Instr. and Meth. A 355 (1995) 386.

[8] M. Campbell et al. (RD-19 Collaboration), Nucl. Instr. and Meth. A 342 (1994) 52.

[9] T. Gys, "Position-sensitive single-photon detection with a hybrid silicon pixel array tube", to appear in Proc. of 1st Conf. on Developments in Photodetection, Beaune (June 1996).

[10] E.H. Darlington, J. Phys. D 8 (1975) 85.

[11] E.H.M. Heijne et al., CERN-ECP/96-03, presented at Hiroshima Symposium (1995), submitted to Nucl. Instr. and Meth. A.

[12] P. Weilhammer, "Performance of silicon pad sensors for RICH detectors", these proceedings, presented at BEAUTY 96, Rome (June 1996). 